\section{Neue Wege gehen}

Bildgebende Verfahren und interventionelle Therapien sind wesentliche Motoren des medizinischen Fortschritts. Die Radiologie ist mit ihrer Vielfalt, ihrer Innnovationsbereitschaft und ihrem Blick für das medizinische Ganze an der Spitze der Entwicklungen, wenn neue Wege in Diagnostik und Behandlung der uns anvertrauten Patienten beschritten werden.

Der Deutsche Röntgenkongress ist Ausdruck der Stärke und Bereitschaft unseres Fachs, sich immer wieder auf neue Wege zu begeben. Beim 97. Deutschen Röntgenkongress 2016 in Leipzig wird dies in besonderer Weise in 4 Schwerpunktthemen deutlich, die - neben allen anderen Bereichen der Radiologie - besonders hervorgehoben werden.

Fast jeder von uns leidet mindestens $1 \mathrm{Mal}$ in seinem Leben unter Rückenschmerzen. Erkrankungen der Wirbelsäule sind eine multidisziplinäre medizinische Herausforderung mit hoher volkswirtschaftlicher, aber auch sozial- und arbeitsmedizinischer Relevanz. Bildgebung und Interventionen tragen wesentlich dazu bei, die vielfältigen somatischen Ursachen von Wirbelsäulenerkrankungen zu erkennen und therapeutisch zu beeinflussen. Dies gilt insbesondere bei Traumafolgen sowie entzündlichen und tumorösen Veränderungen, aber auch bei altersbedingten und degenerativen Pathologien. Gleichzeitig muss sich die Radiologie gerade beim unspezifischen Rückenschmerz auch etwas zurücknehmen, weil Bildgebung eine Reihe von Ursachen eben nicht abbilden kann. Es ist unsere Aufgabe, unsere Werkzeuge zum richtigen Zeitpunkt einzusetzen, aber auch in bestimmten Situationen bildgebende Diagnostik zu unterlassen.

Der radiologische Impact in der Mammadiagnostik ist unbestritten hoch. Diagnostik und Therapie des Mammakarzinoms sind in den letzten 10 Jahren kontinuierlich besser geworden. Neue Wege werden durch technische Innovationen der Bildgebung und durch individualisierte Tumortherapien beschritten. Noch vor einigen Jahren hätten wir uns kaum vorstellen können, dass Röntgen-Schichtbildverfahren die mammografische Diagnostik weiter nach vorne bringen könnten. Beim Monitoring individualisierter neoadjuvanter Systemtherapien, die bei manchen Patientinnen mit Mammakarzinom geradezu spektakuläre Erfolge zeigen, ist die Mamma-Radiologie inzwischen unverzichtbar. Radiologen sind kompetente Partner in Teams, die das Mammakarzinom als biologische Herausforderung und nicht nur als lokales Problem verstehen. Als Teil des Programms zur Krebsfrüherkennung bei Frauen steht das Mammografie-Screening inzwischen auf so breiter Basis, dass in 2016 erste messbare epidemiologische Effekte erwartet werden.

Mit der Wiederentdeckung der Stärken des Ultraschalls in der Radiologie beschreitet unser Fach sozusagen „neue alte Wege“. Der bildgebende Ultraschall hat seine historischen Wurzeln in unserer Disziplin. In Rückbesinnung auf diese Tradition implementieren wir neue Techniken wie die kontrastmittelverstärkte Sonografie (CEUS) und die Elastografie in unsere diagnostischen Konzepte. Mit durch Ultraschall gesteuerten Interventionen erweitern wir unser minimalinvasives Spektrum. Die Radiologie deckt damit Bildgebung und Interventionen mit ihren morphologischen und funktionellen Facetten integral ab, weil wir alle bildgebenden Verfahren situationsgerecht, ergebnisorientiert, schonend und wirtschaftlich einsetzen können. Wir dürfen die Sonografie nicht anderen Disziplinen überlassen, sondern müssen auch hier immer kompetenter werden und Innnovationen vorantreiben.

Der Umgang mit Fehlern und Komplikationen ist in der Medizin transparenter und ehrlicher geworden. Die Radiologie ist ein

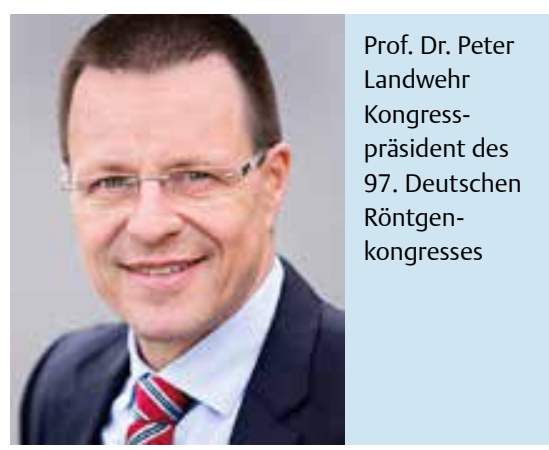

technikaffines Fach, das von Menschen betrieben wird. Technik und Menschen sind nie fehlerfrei. Dies offen anzusprechen und sich mit seinen Fehlern zu beschäftigen, sind erste und wichtige Schritte, um in Zukunft noch weniger Fehler und damit die Radiologie noch sicherer zu machen.

Diese und viele weitere Themen werden kompetente Referenten, aktive Kolleginnen und Kollegen in diversen Workshops sowie die Teilnehmer des 97. Deutschen Röntgenkongresses vom 4.-7. Mai 2016 in Leipzig diskutieren. Neben der inhaltlichen Weiterentwicklung wird auch der Deutsche Röntgenkongress neue Wege gehen: mit Veranstaltungsformaten, die vermehrt das fallbasierte und interaktive Lernen in den Vordergrund stellen, mit einer großen Anzahl Hands-On-Workshops, einer weiterentwickelten RöKoApp und mit einer nun zentralen Industrieausstellung, die den Austausch zwischen Nutzern und Herstellern befördert.

Freuen Sie sich auf einen neuen, modernen Kongressort in einer traditionsreichen Messestadt voller Kultur und Geschichte! Leipzig bietet genau die richtige Mischung, um jeden von uns für neue Wege in der Radiologie zu begeistern.

\section{Prof. Dr. Peter Landwehr}

Kongresspräsident des 97. Deutschen Röntgenkongresses

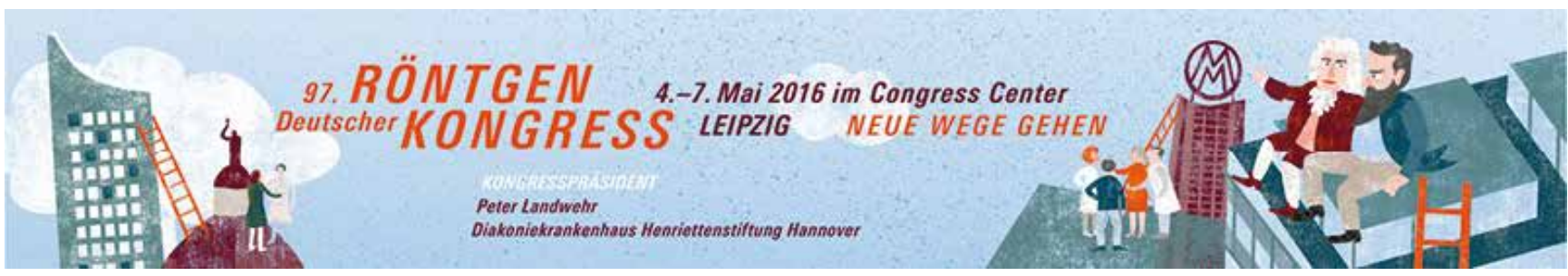

